\title{
A therapy that'll put hair in your ears
}

A new gene therapy approach has led to the first major success story in the ongoing effort to develop techniques countering deafness due to inner ear damage.

Highly specialized epithelial cells known as 'hair cells', located within an inner-ear structure called the cochlea, have a central role in the mammalian auditory system, in that they are directly involved in translating the vibrations from sound waves into signals that can be interpreted by the brain. Damage to these cells can have a profound effect on hearing, and degeneration and loss of hair cells - from causes both natural and unnatural-is among the more common reasons for onset of deafness. Importantly, the body does not replace lost hair cells on its own, leaving the onus on researchers to develop strategies for the restoration of these valuable cells.

A group of American and Japanese researchers, led by Yehoash Raphael at the University of Michigan (Ann Arbor), have stepped up to the challenge, focusing their attention on Atoh1, a gene known to be important in the differentiation of hair cells during embryonic development. Previous studies have indicated that the action of Atoh1 can induce nonsensory cells in the cochlea to generate new hair cells; Raphael and his colleagues now sought to find out whether the viralmediated transfer of this gene to the inner ears of deafened guinea pigs could help restore hair cell growth and hearing (Nat. Med., March).

They chemically deafened the animals in a manner that eliminated virtually all hair cells and then treated the damaged cochleas with an adenoviral vector containing the Atoh1 gene. Effective transduction of the gene into and expression by the nonsensory cells of the cochlea took place, and within 8 weeks, large numbers of hair cells were detectable, with the most effective restoration occurring near the site of injection.

There are two basic types of hair cells: outer cells (OHCs), which amplify vibratory signals, and inner cells (IHCs), which actually convey information to the brain via the auditory nerve. This gene therapy approach proved most effective at IHC restoration, and these cells became fully differentiated and relatively well organized; the
OHCs, on the other hand, generally failed to reach full maturation. Nevertheless, by 8 weeks, the treatment had restored a considerable amount of acoustic sensitivity to the animals, as determined by a commonly used hearing test, and this recovery remained constant for at least several weeks afterward.

The researchers speculate that Atoh1 might trigger mature nonsensory cells in the cochlea to proliferate and redifferentiate as hair cells, or that perhaps the cochlea contains an undiscovered trove of stem cells that respond to this gene. Clearly, much more investigation will be necessary, but these early findings at least offer new promise for the restoration of lost hearing.

Michael Eisenstein

\section{ONE GENE, ONE COMPLEX NEUROLOGICALDISORDER?}

Altered expression of a single gene could prove to be the basis of even seemingly complex mental illnesses, according to microarray analyses of rat brains. These results may help explain the origins of schizophrenia in humans.

There is a consensus that schizophrenia, a severe and debilitating brain disorder characterized by hallucinations, delusions, paranoia, erratic thinking, and antisocial behavior, results from a combination of genetic and developmental conditions, but there has been no determination of the exact cause. Approximately $1 \%$ of the population develops schizophrenia during their lifetime and, although antipsychotic medications can relieve many of these symptoms, few people ever achieve a complete cure.

Now, a discovery in rats may help researchers pinpoint the molecular underpinnings of schizophrenia in humans. Gerard J.M. Martens of the Nijmegen Center for Molecular Life Sciences (Nijmegen, The Netherlands) studied rats that had been pharmacologically selected and bred to be either susceptible (APO-SUS) or resistant (APO-UNSUS) to the dopaminergic agonist apomorphine. Compared to their APO-UNSUS counterparts, APO-SUS rats display neuroanatomical, biochemical, and behavioral traits similar to those seen in humans with schizophrenia.
Martens's group performed microarray analyses on the hippocampus of APO-SUS and APO-UNSUS rats to look for variations in gene expression that might explain these phenotypic differences. Surprisingly, the only significant difference was in the expression of $A p h-1 b$, a component of the $\gamma$-secretase enzyme complex that is involved in numerous neurodevelopmental signaling pathways, from neuronal migration, to angiogenesis, to tumor growth (Neuron, 17 February). Behavioral tests on rats grouped by their level of Aph-1b expression showed a gene dosage effect, that the authors attribute to a genomic rearrangement.

Whether these results apply to humans remains to be seen. As Martens tells Lab Animal, "Unlike rats [that have two copies], humans have only one $A p h-1 b$ gene, so a genomic rearrangement event resulting in a gene-dosage effect as observed in the rats seems unlikely to occur in humans. However, at the genetic or expression level there could well be a link between a $\gamma$-secretase component and complex disorders such as schizophrenia." His team is currently analyzing the Aph-1b gene in humans and studying environmental factors that affect the gene's expression level in rats.

Tanja Schub 